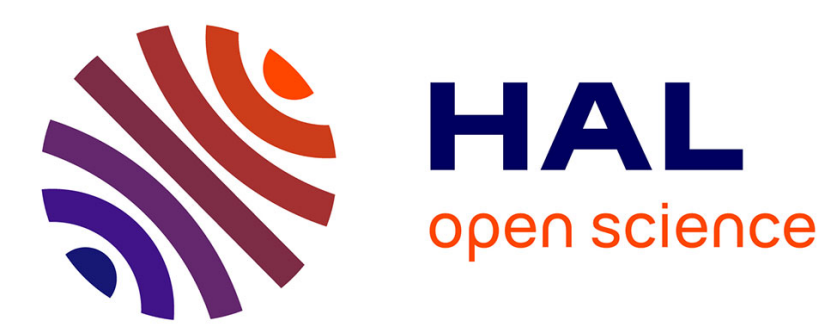

\title{
Connections between Theta-Graphs, Delaunay Triangulations, and Orthogonal Surfaces
}

Nicolas Bonichon, Cyril Gavoille, Nicolas Hanusse, David Ilcinkas

\section{To cite this version:}

Nicolas Bonichon, Cyril Gavoille, Nicolas Hanusse, David Ilcinkas. Connections between ThetaGraphs, Delaunay Triangulations, and Orthogonal Surfaces. WG 2010, Jun 2010, Greece. pp.266-278, 10.1007/978-3-642-16926-7_25. hal-00536710

\section{HAL Id: hal-00536710 https://hal.science/hal-00536710}

Submitted on 16 Nov 2010

HAL is a multi-disciplinary open access archive for the deposit and dissemination of scientific research documents, whether they are published or not. The documents may come from teaching and research institutions in France or abroad, or from public or private research centers.
L'archive ouverte pluridisciplinaire HAL, est destinée au dépôt et à la diffusion de documents scientifiques de niveau recherche, publiés ou non, émanant des établissements d'enseignement et de recherche français ou étrangers, des laboratoires publics ou privés. 


\title{
Connections between Theta-Graphs, Delaunay Triangulations, and Orthogonal Surfaces
}

\author{
Nicolas Bonichon, Cyril Gavoille ${ }^{\star \star}$, Nicolas Hanusse, and David Ilcinkas \\ LaBRI, CNRS \& Université de Bordeaux, France. \\ \{bonichon, gavoille, hanusse, ilcinkas\}@labri.fr
}

\begin{abstract}
Theta_{k}$-graphs are geometric graphs that appear in the context of graph navigation. The shortest-path metric of these graphs is known to approximate the Euclidean complete graph up to a factor depending on the cone number $k$ and the dimension of the space.

TD-Delaunay graphs, a.k.a. triangular-distance Delaunay triangulations, introduced by Chew, have been shown to be plane 2-spanners of the 2D Euclidean complete graph, i.e., the distance in the TD-Delaunay graph between any two points is no more than twice the distance in the plane. Orthogonal surfaces are geometric objects defined from independent sets of points of the Euclidean space. Orthogonal surfaces are well studied in combinatorics (orders, integer programming) and in algebra. From orthogonal surfaces, geometric graphs, called geodesic embeddings can be built.

In this paper, we introduce a specific subgraph of the $\Theta_{6}$-graph defined in the $2 \mathrm{D}$ Euclidean space, namely the half- $\Theta_{6}$-graph, composed of the evencone edges of the $\Theta_{6}$-graph. Our main contribution is to show that these graphs are exactly the TD-Delaunay graphs, and are strongly connected to the geodesic embeddings of orthogonal surfaces of coplanar points in the 3D Euclidean space.

Using these new bridges between these three fields, we establish:

- Every $\Theta_{6}$-graph is the union of two spanning TD-Delaunay graphs. In particular, $\Theta_{6}$-graphs are 2 -spanners of the Euclidean graph, and the bound of 2 on the stretch factor is the best possible. It was not known that $\Theta_{6}$-graphs are $t$-spanners for some constant $t$, and $\Theta_{7}$-graphs were only known to be $t$-spanners for $t \approx 7.562$.

- Every plane triangulation is TD-Delaunay realizable, i.e., every combinatorial plane graph for which all its interior faces are triangles is the TD-Delaunay graph of some point set in the plane. Such realizability property does not hold for classical Delaunay triangulations.
\end{abstract}

Key words: Delaunay triangulation, theta-graph, orthogonal surface, spanner, realizability

* All authors are partially supported by the ANR project "ALADDIN" and the INRIA project "CÉPAGE".

** Member of the "Institut Universitaire de France". Supported by the French-Israeli "Multi-Computing" project. 


\section{Introduction}

A geometric graph is a weighted graph whose vertex set is a set of points of $\mathbb{R}^{d}$, and whose edge set consists of line segments joining two vertices. The weight of any edge is the Euclidean distance $\left(L_{2}\right.$-norm) between its endpoints. The Euclidean complete graph is the complete geometric graph, in which all pairs of distinct vertices are connected by an edge.

Although geometric graphs are in theory specific weighted graphs, they naturally model many practical problems and in various fields of Computer Science, from Networking to Computational Geometry. Delaunay triangulations, Yao graphs, theta-graphs, $\beta$-skeleton graphs, Nearest-Neighborhood graphs, Gabriel graphs are just some of them [17]. A companion concept of geometric graphs is the graph spanner. A $t$-spanner of a graph $G$ is a spanning subgraph $H$ such that for each pair $u, v$ of vertices the distance in $H$ between $u$ and $v$ is at most $t$ times the distance in $G$ between $u$ and $v$. The value $t$ is called the stretch factor of the spanner.

Spanners have been independently introduced in Computational Geometry by Chew [7] for the complete Euclidean graph, and in the fields of Networking and Distributed Computing by Peleg and Ulman [31] for arbitrary graphs. Literature in connection with spanners is vast and applications are numerous. We refer to Peleg's book [30], and Narasimhan and Smid's book [28] for a comprehensive introduction to the topic.

\subsection{Orthogonal surfaces}

With a point set $M$ of $\mathbb{R}^{d}$ it is possible to associate other geometric objects. Assuming that $M$ consists only of pairwise incomparable ${ }^{1}$ points, the orthogonal surface of $M$ is the geometric boundary of the set of points of $\mathbb{R}^{d}$ greater to at least one point of $M$ (see Fig. 2 for $d=3$ ).

Orthogonal surfaces are rich mathematical objects with connections to various fields, including order dimension, integer programming, and monomial ideals. Schnyder woods and orthogonal surfaces of coplanar points of $\mathbb{R}^{3}$ have been established by Miller [27], and Felsner and Zickfeld [15]. As a side effect, they gave an intuitive proof of a restricted version of the Brightwell-Trotter Theorem, which is an extension to multigraphs of Schnyder's characterization of planar graphs in terms of dimension of their incidence order [33].

The geodesic embedding of a point set $S \subset \mathbb{R}^{2}$ is a geometric graph with vertex set $S$. To define its edges, one considers a specific embedding $\phi: S \rightarrow \mathbb{R}^{3}$ such that the points of $\phi(S)$ are coplanar (see Section 2). There is an edge between the points $p, q \in S$ if the join point $\phi(p) \vee \phi(q)$ belongs to the orthogonal surface of $\phi(S)$, the join point being the point with maximum coordinates between $\phi(p)$ and $\phi(q)$ in each dimension.

\footnotetext{
${ }^{1}$ A point $v$ is greater than $u$ if, for each dimension $i, v$ 's $i$ th coordinate is greater than $u$ 's $i$ th coordinate.
} 


\subsection{Delaunay-graphs}

In his seminal paper [7], Chew has constructed plane spanners of the 2D Euclidean graph, namely planar subgraphs whose stretch factor is at most $\sqrt{10} \approx$ 3.162. His construction is based on the $L_{1}$-Delaunay graph, i.e., the dual of the Voronoi diagram for the Manhattan distance ( $L_{1}$-norm). He conjectured that $L_{2}$-Delaunay graphs, i.e., classical Delaunay triangulations, are $t$-spanners for some constant $t$. This conjecture has been proved in [12], and the current best bounds on the stretch factor $t$ of $L_{2}$-Delaunay graphs are $1.584<t<2.419$, proved respectively in [5] and [23]. Determining the exact stretch factor of this important class of geometric graphs is a challenging and open question. We refer to the recent survey [6].

More generally, for any given convex set $\Gamma$ in the plane ${ }^{2}$, one can define the $\Gamma$-Delaunay graphs as the dual of the Voronoi diagram of a set of points with respect to the convex distance function defined by $\Gamma$. Bose et al. [2] have shown that $\Gamma$-Delaunay graphs are plane $t$-spanners for some stretch factor $t$ depending on the shape of $\Gamma$.

A natural question, widely open, is to determine whether $L_{2}$-Delaunay graphs are the "best" plane spanners in terms of stretch factor. It is known [13] that there are point sets for which no plane $t$-spanner can exist if $t<\left(1+10^{-11}\right) \pi / 2 \approx$ 1.570. On the upper bound side, Chew introduced in [7] the triangular distanceDelaunay graphs, TD-Delaunay graphs for short, whose convex distance function is defined from an equilateral triangle. He proved that TD-Delaunay graphs are plane 2-spanners. The stretch 2 is optimal with respect to the triangular distance because of some 3 -gons.

\subsection{Delaunay realizability}

Searching for the "best" plane spanner should be done, a priori, in the set of all planar graphs. Indeed, there is no advantage to limit the search to any restricted subclass, except maybe to plane triangulations. By plane triangulation, we mean a combinatorial plane graph in which all interior faces are triangles ${ }^{3}$. However, there are notorious plane triangulations that cannot be obtained from any $L_{2^{-}}$, Delaunay graphs, e.g., a $K_{4}$ for which a degree- 3 vertex is added in each of its three interior faces $[11,25]$. This leads to the question of realizability of plane triangulations by $L_{2}$-Delaunay graphs, and more generally by $\Gamma$-Delaunay graphs for a convex distance function $\Gamma$. More formally, a plane triangulation $G$ is $\Gamma$ Delaunay realizable if there exists a point set $S$ such that the $\Gamma$-Delaunay graph of $S$ is isomorphic to $G$.

Every triangulation of any polygon, i.e., every maximal outerplane graph, can be realized by a $L_{2}$-Delaunay graph [11]. Based on 3D hyperbolic geometry, Hodgson et al. [19] gave a combinatorial characterization of the graphs that are

\footnotetext{
$\overline{2}$ To be more precise, $\Gamma$ must be a compact and convex set with non-empty interior that contains its origin.

${ }^{3}$ Note that the outer face may not be necessarily a triangle nor the convex hull of the point set, see Figure 1(b).
} 
$L_{2}$-Delaunay realizable, leading to a polynomial-time recognition algorithm by the use of integer programming. The algorithm has been simplified later in [18, 26]. Other connections between toughness, polyhedra of inscribable type, and $L_{2^{-}}$ Delaunay graphs have been developed in [10]. For an arbitrary convex distance function $\Gamma$, the $\Gamma$-Delaunay realizability has not yet been studied.

\subsection{Theta-graphs}

Theta-graphs $[9,22]$ and Yao graphs [34] are very popular geometric graphs that appear in the context of navigating graphs. Adjacency is defined as follows: the space around each point $p$ is decomposed into $k \geqslant 2$ regular cones, each with apex $p$, and a point $q \neq p$ of a given cone $C$ is linked to $p$ if, from $p, q$ is the "nearest" point in $C$. When the points are in general positions, the out-degree is at most $k$, and the points form a non-plane graph in general whenever $k>6$.

Theta-graphs and Yao graphs differ in the way the nearest neighbor is defined. We focus on the 2D Euclidean space, so that each cone forms an angle of $\Theta_{k}=$ $2 \pi / k$. For Yao graphs ( $Y_{k}$-graphs for short), the nearest neighbor of $p$ in the cone $C$ is simply a point $q \neq p$ minimizing the $L_{2}$-distance between $p$ and $q$. Whereas for theta-graphs ( $\Theta_{k}$-graphs for short), the nearest neighbor of $p$ is the point whose orthogonal projection onto the bisector of $C$ minimizes the $L_{2}$-distance.

Both graphs are known to be efficient spanners. The stretch factor of $\Theta_{k^{-}}$ graphs and $Y_{k}$-graphs, proved respectively in [32] and in [34], is at most $1 /(1-$ $2 \sin (\pi / k))$ for every $k>6$. Very little is known for $k \leqslant 6$. For instance, it was known that $Y_{4}$-graphs are connected [16] and recently it has been shown that they are $8(29+23 \sqrt{2})$-spanners ${ }^{4}$ [4]. A very recent result [29] states that $Y_{6}$ graphs are 20.4-spanners. For $k=7$, we observe that the current upper bound on the stretch of these graphs is larger than 7.562, and the upper bound drops under 2 only from $k \geqslant 13$.

Our main result relies on a specific subgraph of the $\Theta_{k}$-graph, namely the half- $\Theta_{k}$-graph, taking only half the edges, those belonging to non-consecutive cones in the counter-clockwise order (see Section 2 for a more formal definition). For even $k$, every $\Theta_{k}$-graph is the union of two spanning half- $\Theta_{k}$-graphs.

\subsection{Our results}

Our main contribution is an unexpected connection between theta-graphs, TDDelaunay graphs, and orthogonal surfaces. We stress that these objects come from rather different domains and can lead to new results. We show that (see Section 3 for a more precise statement):

For every point set $S \subset \mathbb{R}^{2}$ in general position, the half- $\Theta_{6}$-graph of $S$, the TD-Delaunay graph of $S$, and the geodesic embedding of $S$ are equal.

Half- $\Theta_{6}$-graph turns out to be a key ingredient of our result. This unification result implies that each of these objects can directly inherit of all the known properties from the others. In particular, we exhibit two important corollaries:

\footnotetext{
4 This number is greater than 492 .
} 
1. Every $\Theta_{6}$-graph is the union of two spanning TD-Delaunay graphs.

In particular, $\Theta_{6}$-graphs are 2-spanners of the 2D Euclidean graph, because they contain a TD-Delaunay graph as spanning subgraph, which is a 2-spanner from [7]. Since the bound of 2 is optimal (by considering the apices of a quasiequilateral triangle), we have therefore determined the stretch factor of $\Theta_{6^{-}}$ graphs. Up to now, $\Theta_{6}$-graphs were not known to be $t$-spanners for any constant $t$, and the best known bound on the stretch factor for $\Theta_{7}$-graphs was larger than 7.562. Before this current paper, only $\Theta_{k}$-graphs for $k \geqslant 13$ were known to be 2 -spanners (see the previous best general upper bound [32]).

The other important consequence is:

2. Every plane triangulation is TD-Delaunay realizable.

We also show that the plane triangulation of $K_{4}$ is not $L_{1}$-Delaunay realizable, so that, to the best of our knowledge, the equilateral triangle is the only regular convex distance function $\Gamma$ that is known to have the property that every plane triangulation is $\Gamma$-Delaunay realizable.

The paper is organized as follows. In Section 2 we precisely define all the objects we need, and in Section 3 we prove our main result. The corollaries are proved in Section 4. Due to space limitations, proofs are omitted.

\section{Definitions}

\section{$2.1 \quad$ Half- $\Theta_{6}$-graph}

A cone is the region in the plane between two rays that emanate from the same point, its apex. For each cone $C$, let $\ell_{C}$ be the bisector ray of $C$, and for each point $p$, let $C^{p}=\{x+p: x \in C\}$. Let us consider the rays obtained by a counter-clockwise rotation around the origin of the positive $x$-axis by angles of $2 i \pi / k$ with integer $i$. Each pair of successive rays $2(i-1) \pi / k$ and $2 i \pi / k$ defines a cone, denoted by $A_{i}$, whose apex is the origin. Let $\mathcal{A}_{k}=\left\{A_{1}, \ldots, A_{k}\right\}$.

The directed $\Theta_{k}$-graph of a point set $S \subset \mathbb{R}^{2}$, denoted by $\overrightarrow{\Theta_{k}}(S)$, is defined as follows: (1) the vertex set of $\overrightarrow{\Theta_{k}}(S)$ is $S$; and $(2)(p, r)$ is an $\operatorname{arc}$ of $\overrightarrow{\Theta_{k}}(S)$ if and only if there is a cone $A_{i} \in \mathcal{A}_{k}$ such that $r \in A_{i}^{p} \backslash\{p\}$ whose orthogonal projection onto $\ell_{C}^{p}$ is the closest to $p$.

This definition makes no assumptions on relative positions of points of $S$. In particular, it may happen that in $\overrightarrow{\Theta_{k}}(S)$ several arcs of a given cone have the same length (and the out-degree is larger than $k$ ), or some arc lie on the border of a cone. The notion of "general position" is discussed in Section 3. We now introduce a new graph, called half- $\Theta_{k}$-graph, defined as follows:

Definition 1. The directed half- $\Theta_{k}$-graph of a point set $S \subset \mathbb{R}^{2}$, denoted by $\frac{1}{2} \overrightarrow{\Theta_{k}}(S)$, is the digraph induced by all the arcs $(p, r)$ of $\overrightarrow{\Theta_{k}}(S)$ such that $r \in A_{i}^{p}$ for some even number $i$.

Whenever $k \equiv 2(\bmod 4)$, we denote by $C_{i}$ the cone $A_{2 i}$, and by $\bar{C}_{i}$ the opposite cone of $C_{i}$, i.e., $\bar{C}_{i}=A_{2 i+k / 2 \bmod 6}$ (observe that $2 i+k / 2$ is odd). An arc $(p, r)$ such that $r \in C_{i}^{p}$ is said to be colored $i$. 


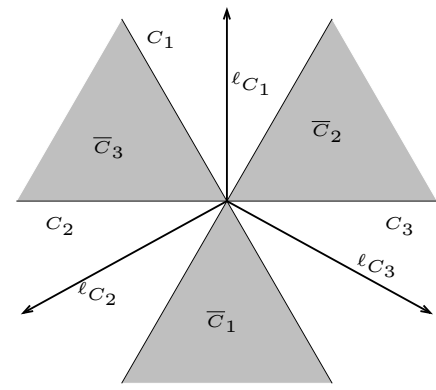

(a)

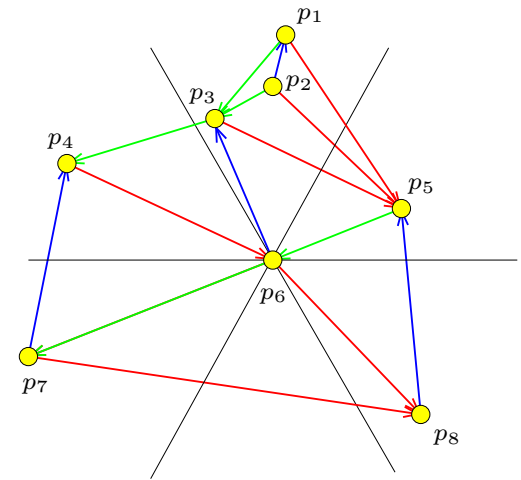

(b)

Fig. 1. (a) Illustration of notations for half- $\Theta_{6}$-graphs. (b) An example of a directed half- $\Theta_{6}$-graph.

In this paper, we focus on the half- $\Theta_{6}$-graph. So, in counter-clockwise order starting from the positive $x$-axis, the six cones of $\mathcal{A}_{6}$ are encountered in the order $\bar{C}_{2}, C_{1}, \bar{C}_{3}, C_{2}, \bar{C}_{1}, C_{3}$ (see Fig. 1(a)). Fig. 1(b) shows an example of a directed half- $\Theta_{6}$-graph on 8 points.

The set of points $S$ is said to be degenerate if there exist two points $p$ and $q$ in $S$ such that both $(p, q)$ and $(q, p)$ are $\operatorname{arcs}$ of $\frac{1}{2} \overrightarrow{\Theta_{k}}(S)$. The set $S$ is said to be non-degenerate otherwise.

\subsection{Geodesic embeddings}

Let $\mathcal{P}$ be a plane equipped with the standard basis $\left(\mathbf{e}_{\mathbf{x}}, \mathbf{e}_{\mathbf{y}}\right)$, and let $S$ be a finite set of points in the plane $\mathcal{P}$.

The following definitions are extracted from [27]. (Similar definitions can also be found in Felsner's book [14].) Let $\left(\mathbf{e}_{\mathbf{1}}, \mathbf{e}_{\mathbf{2}}, \mathbf{e}_{\mathbf{3}}\right)$ be the standard basis of $\mathbb{R}^{3}$. The plane $\mathcal{P}$ is now embedded in $\mathcal{P}^{\prime} \subset \mathbb{R}^{3}$ where $\mathcal{P}^{\prime}$ is the plane containing the origin of $\mathbb{R}^{3}$ with basis $\left(\mathbf{e}_{\mathbf{x}}^{\prime}, \mathbf{e}_{\mathbf{y}}^{\prime}\right)$ where $\mathbf{e}_{\mathbf{x}}^{\prime}=(0,-1 / \sqrt{2}, 1 / \sqrt{2})$ and $\mathbf{e}_{\mathbf{y}}^{\prime}=$ $(\sqrt{2 / 3},-1 / \sqrt{6},-1 / \sqrt{6})$. Observe that $\mathbf{e}_{1}+\mathbf{e}_{2}+\mathbf{e}_{3}$ is a normal vector ${ }^{5}$ of $\mathcal{P}^{\prime}$. Any point $p=\left(p_{x}, p_{y}\right) \in \mathbb{R}^{2}$ is mapped to $p^{\prime} \in \mathcal{P}^{\prime}$ with $p^{\prime}=p_{x} \mathbf{e}_{\mathbf{x}}^{\prime}+p_{y} \mathbf{e}_{\mathbf{y}}^{\prime}$.

Consider the dominance order on $\mathbb{R}^{3}: p \succcurlyeq q$ if and only if $p_{i} \geqslant q_{i}$ for each $i \in\{1,2,3\}$. Note that any two different points of $\mathcal{P}^{\prime}$ are incomparable. The filter generated by a set of points $S$ of $\mathcal{P}$ is the set

$$
\langle S\rangle=\left\{\alpha \in \mathbb{R}^{3}: \alpha \succcurlyeq v \text { for some } v \in S\right\} .
$$

The boundary $\mathfrak{S}_{S}$ of $\langle S\rangle$ is the coplanar orthogonal surface generated by $S$. Notice that in $[27,15]$, the authors also consider orthogonal surfaces, a more general case where elements of $S$ are pairwise incomparable but not necessarily

\footnotetext{
${ }^{5}$ I.e., $\forall p^{\prime}=\left(p_{1}^{\prime}, p_{2}^{\prime}, p_{3}^{\prime}\right) \in \mathcal{P}^{\prime}, p_{1}^{\prime} \mathbf{e}_{1}+p_{2}^{\prime} \mathbf{e}_{2}+p_{3}^{\prime} \mathbf{e}_{3}=0$.
} 


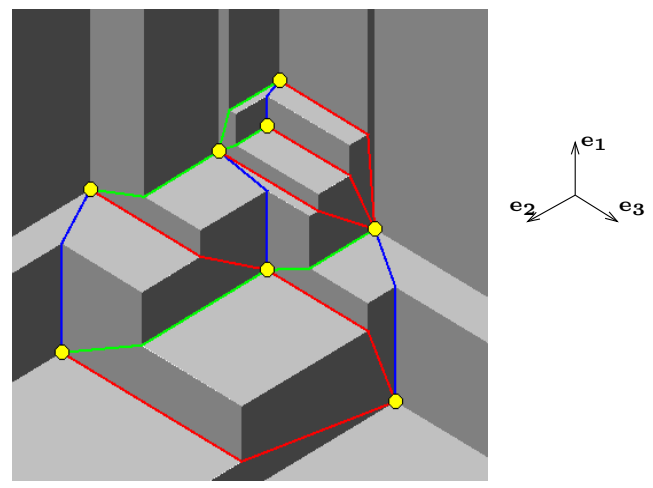

Fig. 2. A coplanar orthogonal surface with its geodesic embedding.

in the same plane of normal vector $\mathbf{e}_{1}+\mathbf{e}_{2}+\mathbf{e}_{3}$. Fig. 2 shows an example of a coplanar orthogonal surface.

We denote by $p \vee q$ the point $\left(\max \left\{p_{1}, q_{1}\right\}, \max \left\{p_{2}, q_{2}\right\}, \max \left\{p_{3}, q_{3}\right\}\right)$. If $p, q \in$ $S$ and $p \vee q \in \mathfrak{S}_{S}$, then $\mathfrak{S}_{S}$ contains the union of the two line segments joining $p$ and $q$ to $p \vee q$. These lines are called elbow geodesics of $\mathfrak{S}_{S}$. The orthogonal arc of $p \in S$ in direction of the standard vector $\mathbf{e}_{\mathbf{i}}$ is the piece of ray $p+\lambda \mathbf{e}_{\mathbf{i}}, \lambda \geqslant 0$, which follows a crease of $\mathfrak{S}_{S}$. If $p \vee q$ is equal to $p+\lambda \mathbf{e}_{\mathbf{i}}$, for some $\lambda \geqslant 0$, we say that it is an elbow of type $i$. The corresponding elbow geodesic is also said to be of type $i$. Observe that $p \vee q$ shares two coordinates (on the basis $\left(\mathbf{e}_{\mathbf{1}}, \mathbf{e}_{\mathbf{2}}, \mathbf{e}_{\mathbf{3}}\right)$ ) with at least one (and perhaps both) of $p$ and $q$. We say that a geodesic elbow is uni-directed if its corresponding elbow $p \vee q$ shares two of its coordinates either with $p$ or with $q$ (but not with both).

An orthogonal surface $\mathfrak{S}_{S}$ is uni-directed if all the geodesic elbows are unidirected.

Definition 2. Let $S$ be a set of points on $\mathcal{P}$ such that the orthogonal surface $\mathfrak{S}_{S}$ is uni-directed. The geodesic embedding of $S$ is the directed graph $\overrightarrow{\mathbf{G e o}}(S)$ defined as follows:

- the vertices of $\overrightarrow{\mathbf{G e o}}(S)$ are the points of $S$.

- there is an arc from $p$ to $q$ colored $i$ if and only if $p \vee q$ is an elbow of type $i$.

\subsection{TD-Delaunay triangulation}

We recall here the definition of TD-Delaunay graphs introduced in [7].

Let $T$ (resp. $\tilde{T}$ ) be the equilateral triangle of side length 1 whose barycenter is the origin and one of its vertices is on the positive (resp. negative) $y$-axis . A homothet of $T$ is obtained by scaling $T$ with respect to the origin, followed by a translation: $p+\lambda T=\{p+\lambda z: z \in T\}$. The triangular distance between two points $p$ and $q$ is defined as follows:

$$
d_{T}(p, q)=\min \{\lambda: \lambda \geqslant 0 \text { and } q \in p+\lambda T\}
$$


Notice that in general $d_{T}(p, q) \neq d_{T}(q, p)$.

Let $S$ be a set of points in the plane $\mathcal{P}$. For each $p \in S$, we define the $T D$-Voronoi cell of $p$ as:

$$
V_{T}(p)=\left\{x \in \mathcal{P}: \text { for all } q \in S, d_{T}(p, x) \leqslant d_{T}(q, x)\right\} .
$$

Fig. 3(a) shows an example of a set of TD-Voronoi cells, also called TD-Voronoi diagram. Observe that the intersection of two TD-Voronoi cells may have a positive area. For instance, consider the following set $S=\{u=(-\sqrt{3}, 1), v=(\sqrt{3}, 1)\}$ (see Fig. 3(b)). The intersection $V_{T}(u) \cap V_{T}(v)$ contains the part of the plane below the two lines $(o, u)$ and $(o, v)$ where $o=(0,0)$.
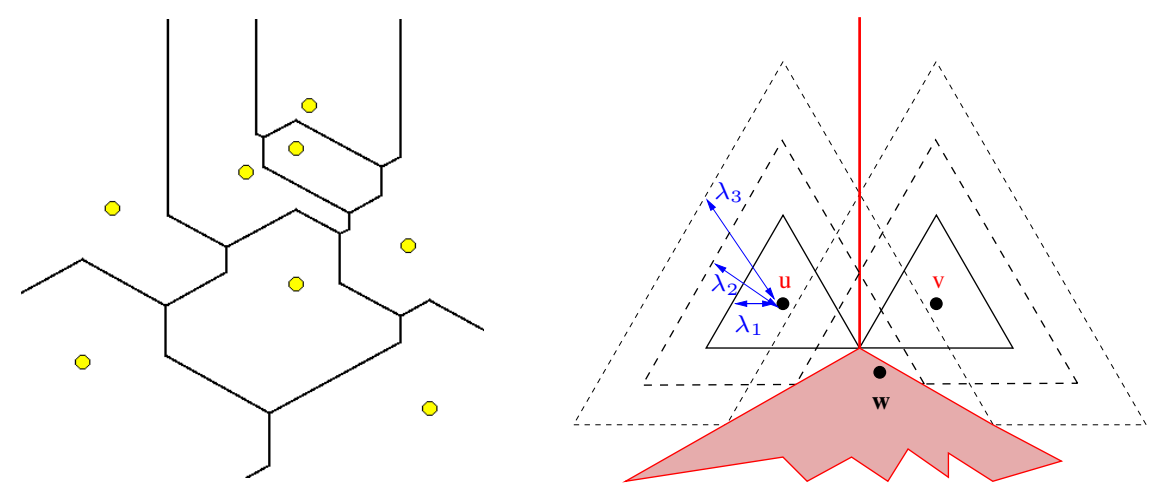

Fig. 3. (a) TD-Voronoi diagram. (b) $\lambda_{1}<\lambda_{2}<\lambda_{3}$ stand for three triangular distances. The set $\{u, v\}$ is an ambiguous point set, however $\{u, v, w\}$ is non-ambiguous.

We say that a set of points $S$ is non-ambiguous if the intersection of any two TD-Voronoi cells of $S$ is of null area ${ }^{6}$.

Definition 3. Let $S$ be a non-ambiguous set of points of $\mathcal{P}$. The TD-Delaunay graph of $S$, denoted by $\operatorname{TDDel}(S)$, is defined as follows:

- the vertex set of $\mathbf{T D D e l}(S)$ is $S$; and

$-(p, q)$ is an edge of $\mathbf{T D D e l}(S)$ if and only if $V_{T}(p) \cap V_{T}(q) \neq \varnothing$.

\section{$3 \quad$ Unification of the concepts}

We will now prove that the three objects defined in Section 2 are essentially the same. Note that Fig. 1(b), 2, and 3(a) are based on the same set of points.

Given two points $p$ and $q \in C_{i}^{p}$, we denote by $T_{i}(p, q)$ the set of points of $\mathcal{P}$ in $C_{i}^{p} \backslash\{p\}$ whose orthogonal projection onto $\ell_{C_{i}^{p}}$ is strictly closer to $p$ than the

\footnotetext{
${ }^{6}$ For ambiguous set of points $S$, it is possible to have a partition of the plane by the interior of Voronoi cells plus the union of all boundaries, by ordering the elements of $S$ to break ties. See for example [2].
} 
orthogonal projection onto $\ell_{C_{i}^{p}}$ of $q$. Note that the boundary of $T_{i}(p, q)$ is an equilateral triangle. The interior of $T_{i}(p, q)$ is denoted by $T_{i}^{\circ}(p, q)$. Differently speaking, $T_{i}^{\circ}(p, q)$ is the set of points $T_{i}(p, q)$ deprived of the points lying on the axes of the cone $C_{i}^{p}$.

Lemma 1. Let $S$ be a set of points in the plane $\mathcal{P}$, and let $p$ and $q$ be two distinct points in this set. There is an arc $(p, q)$ colored $i$ in $\frac{1}{2} \overrightarrow{\Theta_{6}}(S)$ if and only if $q \in C_{i}^{p}$ and $T_{i}(p, q) \cap S=\varnothing$.

Lemma 2. Let $S$ be a set of points in the plane $\mathcal{P}$, and let $p$ and $q$ be two distinct points in this set. $p \vee q$ is an elbow of type $i$ if and only if $q \in C_{i}^{p}$ and $T_{i}^{\circ}(p, q) \cap S=\varnothing$.

Lemma 3. Let $S$ be a set of points in the plane $\mathcal{P}$, and let $p$ and $q$ be two distinct points in this set. The Voronoi cells $V_{T}(p)$ and $V_{T}(q)$ share at least a point if and only if there exists $i \in\{1,2,3\}$ such that $q \in C_{i}^{p}$ and $T_{i}^{\circ}(p, q) \cap S=\varnothing$, or $p \in C_{i}^{q}$ and $T_{i}^{\circ}(q, p) \cap S=\varnothing$.

Thanks to these technical lemmas, we first show the links existing between the different notions of "general position" corresponding to the three objects into consideration, and we then prove our main equivalence theorem.

Theorem 1. Let $S$ be a set of points in the plane $\mathcal{P}$.

1. $S$ is non-degenerate if and only if $\mathfrak{S}_{S}$ is uni-directed.

2. If $S$ is non-degenerate, then $S$ is non-ambiguous.

Theorem 2. Let $S$ be a non-degenerate point set in the plane $\mathcal{P}$. Let $\mathbf{G e o}(S)$, resp. $\frac{1}{2} \Theta_{6}(S)$, be the underlying undirected uncolored graph of $\overrightarrow{\mathbf{G e o}}(S)$, resp. $\frac{1}{2} \overrightarrow{\Theta_{6}}(S)$. We have

Moreover,

$$
\frac{1}{2} \Theta_{6}(S)=\mathbf{G e o}(S)=\mathbf{T D D e l}(S)
$$

$$
\overrightarrow{\mathbf{G e o}}(S)=\frac{1}{2} \overrightarrow{\Theta_{6}}(S)
$$

\section{Applications}

\subsection{Spanner}

In [7] it is shown that TD-Delaunay triangulations are plane 2-spanners. From Theorem 2, while observing that the $\Theta_{6}$-graph is the union of two half- $\Theta_{6}$-graphs (one using odd cones and the other even cones) we directly get the following corollary:

Corollary 1. Every half- $\Theta_{6}$-graph (and also $\Theta_{6}$-graph) is a 2-spanner. Moreover the edges of the $\Theta_{6}$-graph can be partitioned into two planar graphs.

We observe that the bound of 2 is the best possible stretch for $\Theta_{6}$-graphs and half- $\Theta_{6}$-graphs. Indeed, the $\Theta_{6}$-graph, and the half- $\Theta_{6}$-graph as well, of some 3 -gons (the apex of a quasi-equilateral triangle) has stretch arbitrarily close to 2 . 


\subsection{Delaunay realizability}

Using the face counting algorithm introduced by Schnyder [33], Felsner and Zickfeld [15, Theorem 10] showed that for every plane triangulation $G$, a point set $S$ such that $\operatorname{Geo}(S)=G$ can be computed in linear time ${ }^{7}$. Using the equivalence between geodesic embeddings and TD-Delaunay triangulations (Theorem 2) we directly get the following result (see Section 1.3 for the definition of realizability):

Corollary 2. Every plane triangulation is TD-Delaunay realizable.

This raises the following natural question: is there another distance function $\Gamma$ such that every triangulation is $\Gamma$-Delaunay realizable? The first natural distance to be considered is the $L_{1}$-norm. The next theorem shows that not all triangulations are $L_{1}$-Delaunay realizable.

Theorem 3. The plane triangulation of $K_{4}$ is not $L_{1}$-Delaunay realizable.

To conclude on realizability, let us mention, that there are graphs that are realizable for a certain distance function and not for another and vice versa. For instance, Theorem 3 shows that $K_{4}$ is not $L_{1}$-Delaunay realizable but it is $L_{2}$-Delaunay realizable. On the other hand, there also exist graphs that are $L_{1}$-Delaunay realizable but not $L_{2}$-Delaunay realizable [11, Fig. 4$]$.

\section{Final remarks}

A Voronoi diagram is sometimes interpreted as a view from the top of a collection of cones whose apices lie on a plane and whose axes are oriented downward (see, e.g., [20]). Coplanar orthogonal surfaces are the exact generalisation of this idea for TD-Voronoi diagrams: the only difference lies on the shape of the base of the cones: circular $\left(L_{2}\right.$-norm), square $\left(L_{1^{-}}\right.$and $L_{\infty}$-norm) or triangular (triangular distance). Hence the TD-Voronoi cell of a point $p$ of $S$ is exactly the orthogonal projection on $\mathcal{P}$ of the points of $\mathfrak{S}_{S}$ dominated by $p$ (see Fig. 2 and 3 ).

Among various generalizations of Voronoi diagrams, Additively Weighted Voronoi Diagrams have been widely studied (see, for example, [3, 24,21]). In such a diagram, the point set is replaced by a set of weighted points $S=$ $\left\{\left(p_{1}, w_{1}\right), \ldots,\left(p_{n}, w_{n}\right)\right\}$. The distance between an element $\left(p_{i}, w_{i}\right)$ of $S$ and point $x$ of the plane $\mathcal{P}$ is $d_{A W}\left(\left(p_{i}, w_{i}\right), x\right)=d\left(p_{i}, x\right)-w_{i}$. The AW-Vonoroi cell of a weighted point $\left(p_{i}, w_{i}\right) \in S$ is naturally defined as follows:

$V_{A W}\left(p_{i}, w_{i}\right)=\left\{x \in \mathcal{P}: \forall\left(p_{j}, q_{j}\right) \in S, i \neq j, d_{A W}\left(\left(p_{i}, w_{i}\right), x\right) \leqslant d_{A W}\left(\left(p_{j}, w_{j}\right), x\right)\right\}$

An AW-Voronoi diagram can be interpreted as a view from the top of a collection of cones where the altitude of the apex of a cone is the weight of the corresponding element of $S$.

\footnotetext{
7 Note that that this result holds also for 3 -connected plane maps, but in this case the orthogonal surface is not uni-directed.
} 
In our context we can define the Additively Weighted Triangular Distance Voronoi Diagram (or simply $A W T D$-Voronoi diagram) using the notion of distance: $d_{A W T D}\left(\left(p_{i}, w_{i}\right), x\right)=d_{T}\left(p_{i}, x\right)-w_{i}$. From the previous remarks, one can see orthogonal surfaces (not necessarily coplanar) as AWTD-Voronoi diagrams.

The Yao graph [34] is very similar to the $\Theta$-graph: in each cone of apex $p$, the selected neighbor of $p$ is the nearest one in the cone instead of being the one with the nearest projection on $\ell_{C}$. Half- $Y_{6}$-graphs can be defined as we did for half- $\Theta_{6}$-graphs considering only 3 of the six cones. Unfortunately, half- $Y_{6}$-graphs do not have as nice structural properties. For instance, a half- $Y_{6}$-graph is not a plane graph in general.

Algorithms that compute $\Theta$-graphs, geodesic embeddings and TD-Delaunay triangulations have been respectively proposed in $[28,15,8]$. It appears that the three proposed algorithms have the same time complexity of $O(n \log n)$ and are all essentially based on the "plane-sweep" algorithm. Hence, our connections between these objects do not give immediately a new insight from the algorithmic point of view.

Finally, the results in this paper have been recently used to construct a plane spanner of maximum degree 6 and stretch factor 6 of any Euclidean graph [1].

\section{References}

1. Bonichon, N., Gavoille, C., Hanusse, N., Perković, L.: Plane Spanners of Maximum Degree Six. In: ICALP '10: Proc. 37th Int. Colloquium on Automata, Languages and Programming. vol. 6198 of LNCS, pp. 19-30 (2010)

2. Bose, P., Carmi, P., Collette, S., Smid, M.: On the Stretch Factor of Convex Delaunay Graphs. In: ISAAC '08: Proc. of $19^{\text {th }}$ Annual Int. Symp. on Algorithms and Computation. vol. 5369 of LNCS, pp. 656-667 (2008)

3. Bose, P., Carmi, P., Couture, M.: Spanners of Additively Weighted Point Sets. In: SWAT '08: 11th Scandinavian workshop on Algorithm Theory. pp. 367-377 (2008)

4. Bose, P., Damian, M., Douïeb, K., O'Rourke, J., Seamone, B., Smid, M.H.M., Wuhrer, S.: Pi/2-Angle Yao Graphs are Spanners. CoRR abs/1001.2913 (2010)

5. Bose, P., Devroye, L., Löffler, M., Snoeyink, J., Verma, V.: The spanning ratio of the Delaunay triangulation is greater than $\pi / 2$. In: CCCG '09: Proc. of $21^{\text {st }}$ Canadian Conf. on Computational Geometry (2009)

6. Bose, P., Smid, M.: On plane geometric spanners: a survey and open problems (2009), submitted

7. Chew, L.P.: There are planar graphs almost as good as the complete graph. Journal of Computer and System Sciences 39(2), 205-219 (1989)

8. Chew, P., Drysdale, R.L.: Voronoi diagrams based on convex distance functions. In: SCG '85: Proc. 1st ann. symp. on Computational geometry. pp. 235-244 (1985)

9. Clarkson, K.: Approximation algorithms for shortest path motion planning. In: STOC '87: Proc. 19th ann. ACM symp. on Theory of computing. pp. 56-65 (1987)

10. Dillencourt, M.B., Smith, W.D.: Graph-theoretical conditions for inscribability and Delaunay realizability. Discrete Mathematics 161(1-3), 63-77 (1996)

11. Dillencourt, M.B.: Toughness and Delaunay Triangulations. Discrete and Computational Geometry 5(1), 575-601 (Dec 1990)

12. Dobkin, D.P., Friedman, S.J., Supowit, K.J.: Delaunay graphs are almost as good as complete graphs. Discrete \& Computational Geometry 5(4), 399-407 (Dec 1990) 
13. Dumitrescu, A., Ebbers-Baumann, A., Grne, A., Klein, Rolf an Rote, G.: On the geometric dilation of closed curves, graphs, and point sets. Computational Geometry: Theory and Applications 36(1), 16-38 (Jan 2007)

14. Felsner, S.: Geometric graphs and arrangements. Vieweg (2004)

15. Felsner, S., Zickfeld, F.: Schnyder Woods and Orthogonal Surfaces. Discrete and Computational Geometry 40(1), 103-126 (Jul 2008)

16. Fischer, M., Lukovszki, T., Ziegler, M.: Geometric searching in walkthrough animations with weak spanners in real time. In: ESA '98: Proc. 6th Annual European Symp. on Algorithms. vol. 1461 of LNCS, pp. 163-174 (1998)

17. Goodman, J.E., O'Rourke, J. (eds.): Handbook of discrete and computational geometry. CRC Press, Inc., Boca Raton, FL, USA (1997)

18. Hiroshima, T., Miyamoto, Y., Sugihara, K.: Another Proof of Polynomial-Time Recognizability of Delaunay Graphs. IEICE Transactions on Fundamentals of Electronics, Communications and Computer Sciences E83-A(4), 627-638 (Apr 2000)

19. Hodgson, C.D., Rivin, I., Smith, W.D.: A Characterization of Convex Hyperbolic Polyhedra and of Convex Polyhedra Inscribed in the Sphere. Bulletin of the American Mathematical Society 27(3), 256-251 (Oct 1992)

20. Hoff, III, K.E., Keyser, J., Lin, M., Manocha, D., Culver, T.: Fast computation of generalized Voronoi diagrams using graphics hardware. In: SIGGRAPH '99: Proc. 26th ann. conf. on comp. graphics and interactive techniques. pp. 277-286 (1999)

21. Karavelas, M.I., Yvinec, M.: Dynamic Additively Weighted Voronoi Diagrams in 2D. In: ESA '02: Proc. 10th Ann. Europ. Symp. on Algorithms. pp. 586-598 (2002)

22. Keil, J.M.: Approximating the complete Euclidean graph. In: No. 318 on SWAT 88: 1st Scandinavian workshop on algorithm theory. pp. 208-213 (1988)

23. Keil, J.M., Gutwin, C.A.: Classes of graphs which approximate the complete Euclidean graph. Discrete \& Computational Geometry 7(1), 13-28 (Dec 1992)

24. Lee, D.T., Drysdale, R.L.: Generalization of Voronoi Diagrams in the Plane. SIAM Journal on Computing 10(1), 73-87 (1981)

25. Lenhart, W., Liotta, G.: Drawable and forbidden minimum weight triangulations. In: GD '97: $5^{\text {th }}$ Int. Symp. on Graph Drawing. vol. LNCS 1353, pp. 1-12 (1997)

26. Lillis, K.M., Pemmaraju, S.V.: On the Efficiency of a Local Iterative Algorithm to Compute Delaunay Realizations. In: WEA '07: Proc. 7th Int. Workshop on Experimental Algorithms. vol. 5038 of LNCS, pp. 69-86 (2008)

27. Miller, E.: Planar Graphs as Minimal Resolutions of Trivariate Monomial Ideals. Documenta Mathematica 7, 43-90 (2002)

28. Narasimhan, G., Smid, M.: Geometric Spanner Networks. Cambridge University Press (2007)

29. O'Rourke, J.: The Yao Graph $Y_{6}$ is a Spanner. CoRR abs/1003.3713 (2010)

30. Peleg, D.: Distributed Computing: A Locality-Sensitive Approach. SIAM Monographs on Discrete Mathematics and Applications (2000)

31. Peleg, D., Ullman, J.D.: An optimal synchronizer for the hypercube. SIAM Journal on Computing 18(4), 740-747 (1989)

32. Ruppert, J., Seidel, R.: Approximating the $d$-dimensional complete Euclidean graph. In: 3rd Canadian Conference on Computational Geometry (CCCG). pp. 207-210 (1991)

33. Schnyder, W.: Planar Graphs and Poset Dimension. Order 5, 323-343 (1989)

34. Yao, A.C.C.: On constructing minimum spanning trees in $k$-dimensional spaces and related problems. SIAM Journal on Computing 11(4), 721-736 (1982) 